\title{
Research Paper: \\ The Effects of Music Intensity on Performance and Cardiovascular Responses in Athletes
}

\author{
Paria Torkamandi $^{1}$ (D), Ali Akbarnejad ${ }^{1 *}$ (D) Abbas Ali Gaeini ${ }^{1}$, Ali Gharecheshmei Ghahremanloo ${ }^{1}$
}

1. Department of Sports Physiology, Faculty of Physical Education and Sports Sciences, University of Tehran, Tehran, Iran.

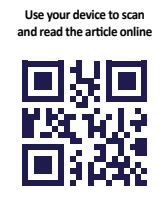

Citation Torkamandi P, Akbarnejad A, Gaeini AA, Gharecheshmei Ghahremanloo A. The Effects of Music Intensity on Performance and Cardiovascular Responses in Athletes Journal of Exercise Science and Medicine. 2020; 12(1):93-100. http:// dx.doi.org/10.32598/JESM.12.1.10

doi) $h$ http://dx.doi.org/10.32598/JESM.12.1.10

\section{(c) (i) (\$)}

Article info:

Received: 19 Oct 2019

Accepted: 05 Nov 2019

Available Online: 01 Jan 2020

Keywords:

Music, Slow music, Blood pressure, Oxygen consumption, Heart rate

\section{ABSTRACT}

Introduction: The current study aimed to evaluate the effects of different musical intensities on performance and cardiovascular responses after incremental exercise in male athletes.

Materials and Methods: In this quasi-experimental study with a cross-over design, 15 male athletes were voluntarily selected. The study subjects performed Bruce protocol, along with listening to progressive music, slow music, and without music until exhaustion.

Results: This study indicated that systolic and diastolic blood pressure, maximum oxygen consumption, maximum heart rate, and time to exhaustion insignificantly decreased while listening to slow music, compared to the no music $(P=0.134, P=0.993, P=0.999, P=0.160$, $\mathrm{P}=0.819$, respectively). Furthermore, while listening to progressive music, compared to no music, the systolic and diastolic blood pressure, as well as maximum heart rate insignificantly increased $(\mathrm{P}=0.735, \mathrm{P}=0.999, \mathrm{P}=0.496$, respectively); the maximum oxygen consumption and the time of exhaustion significantly increased in the study subjects $(P=0.043, P=0.008$ respectively). Moreover, while listening to progressive music, compared to slow music, the systolic blood pressure, maximum oxygen consumption, maximum heart rate, and the time of exhaustion significantly increased $(\mathrm{P}=0.004, \mathrm{P}=0.009, \mathrm{P}=0.002, \mathrm{P}=0.001$ respectively); however, diastolic blood pressure presented an insignificant decrease $(P=0.253)$.

Conclusion: The obtained findings revealed that listening to progressive music can affect physiological factors and performance during exercising. It increases the athlete's motivation and postpones the time to exhaustion to continue exercising; however, listening to slow music creates a state of relaxation during exercise and reduces heart rate. As a result, individuals with hypertension can decline their blood pressure during endurance exercise by listening to soft music.

* Corresponding Author:

Address: Department of Sports Physiology, Faculty of Physical Education and Sports Sciences, University of Tehran, Tehran, Iran. Tel: +98 (912) 0760472

E-mail: aakbarnejad@ut.ac.ir 


\section{Introduction}

articipating in endurance exercises is recommended for athletes and individuals of all ages to improve their health and fitness. However, due to the long time and uniformity performance, fatigue, and pressure during the exercise, their endurance performance decreased [1]. In the last two decades, researches in the field of music psychology have expanded and included various social contexts [2]. A large body of literature suggests that music presents a wide range of psychological effects and acts as an energizing factor. Other researchers suggested that motor function can be facilitated through music in various manners. For example, music can act as a calming or stimulating agent, i.e., effective before or during an activity [2]. Music stimulates the right side of the brain, thereby facilitating cognitive tasks, such as mental practice and visualization [2].

The number of studies exploring musical reactivity and exercise has increased dramatically. Costas Karageorghis and David-Lee Priest, two researchers at Brunel University in London, England, recently published an overview of 62 studies completed since 1997. Part I of this twopart review paper explains the evolution of conceptual approaches and processes underlying music's impact, accompanied by a critical review and synthesis of empirical work (Part II) (spread over Parts I and II). Pre-Task music was demonstrated to increase arousal, facilitate task-relevant visualization, and improve performance in simple motor tasks. Self-selected, motivating, and stimulative music has been manifested to boost impact, minimize the ratings of perceived exertion, improve energy performance, and contribute to improved work output during repetitive, endurance-type tasks. Evidence indicates that carefully chosen music can provide ergogenic and psychological benefits during high-intensity exercise; however, it appears to be unsuccessful in reducing perceived exertion above the anaerobic threshold. The effects of music appear to be at their most potent when it is used to accompany self-paced exercise or in externally valid conditions. The positive effect of music on psychological state and performance is amplified when chosen based on its motivating qualities [3]. If the music conditions are correct, it will influence the wide range of biopsychological changes. This effect is a fundamental question that has attracted the attention of exercise science, medicine, and psychology experts [4].

Multiple researchers have examined the physiological effects and others have assessed the effects of different types of music on physiological variables. For example, it has been stated that fast music increases mental arousal and slow music causes relaxation [5]. Some of the effects of music have been supported to improve physiological conditions in the body such as changes in heart rate, blood pressure, respiration, endorphins levels, skin reactions, brain waves, the limbic nervous system, the control center of the autonomic nervous system, and the reduction of physical pain [6].

Barwood et al. found that participants traveled more distance when running on the treadmill while listening to provocative music, had a lower lactate accumulation rate, and conversely, had an insignificant change in the rate of perceived exertion [7]. Studies revealed that music affects muscle tension, vasoconstriction, immune function, respiration rate, changes in heart rate, and blood pressure [8].

Other studies demonstrated that listening to a variety of music can alter stress hormone levels, including cortisol, adrenaline, and noradrenaline [9]. According to research, music improves motor performance and skills through the mechanisms of reducing fatigue, increasing the levels of arousal, creating harmony, and promoting relaxation and feeling comfortable; all of which are directly related to perceptual processes [8]. However, some of these studies have reached contradictory results on physiological responses that can be due to differences in the types of music, activities, or the conditions of the participants [10].

Birnbaum et al. reported that listening to fast music during exercise significantly increased oxygen consumption, cardiac output, stroke volume, respiratory rate, and minute ventilation; however, there was no significant difference in tidal volume, heart rate, and maximal oxygen consumption [10, 11]. Koc. et al. examined the effects of music on athletes' performance. He noted that music, in general, can provide positive physiological effects (heart rate, blood pressure, body temperature). Furthermore, music, with a fast or slow beat, presents a different effect on performance [12]. Accordingly these results highlight why choosing the right type of music is critical. Moreover, they highlight the significance of the effect of music rhythm on the response to physical activity. Tiev et al. also reported that music significantly reduced the rate of perceived exertion and significantly increased heart rate, ventilation, oxygen consumption, and minute ventilation [13].

Barzegar et al. argued that listening to music during incremental exercise significantly influenced the rate of 
perceived exertion, in the tertiary stage, heart rate, and respiratory exchange in all test stages, $A-\mathrm{VO}_{2}$ Diff in first and second test stages, and the anaerobic threshold. Contrarily, there is no significant difference in blood pressure and fat oxidation between none of the test stages [14]. However, based on the aforementioned researches, it seems complicated to make the right decision to choose the type of music and its impact during sports activities. Therefore, the present study investigated the effects of different intensities of music on the cardiovascular responses after the progressive exercise activities.

\section{Materials and Methods}

This was a quasi-experimental and practical study. Moreover, the statistical population of this study consisted of all young male athlete students of the Faculty of Physical Education, University of Tehran. Fifteen eligible individuals were selected based on the objectives of the study. Accordingly, this group of athletes participated in 3 separate sessions to perform the test. The first session was without music, the second session with slow music, finally the third session with progressive music. In this test, we used a cross-over design (this method was used to eliminate learning and activity effects).

The results related to the quality assessment are illustrated in Table 1. The study participants completed the Bruce test ${ }^{1}$ in three sessions. The first group began with slow music in the first session, continued with slow music in the second session; as the intensity of the exercise grew, so did the intensity of the music (the music began with a beat of $60 \mathrm{bpm}^{2}$ in the early stages of the Bruce test, when the activity intensity reached to the middle stages, the music intensity was increased to a range of $80 \mathrm{bpm}$ to $90 \mathrm{bpm}$, as well. Moreover, when the move-

1. Every 3 min the speed \& incline of the TM are increased. There are 7 such stages and only very fir athletes can complete all 7 stages.

2. Beats per minute

Table 1. Characteristics of subject's (N=15) ment of activity was at its highest intensity -at the final stages of the Bruce test- the number of music' beats exceeded $120 \mathrm{bpm}$. The appropriateness of the bpm was tested using the program (Music 1.5 Adobe Audition) until they worked without music in the third session. The research groups interchanged their places, where each group performed all three types of tests.

The research design consisted of 3 experimental conditions (slow music, progressive music, no-music) in the Physiology Laboratory of the Faculty of Physical Education at the University of Tehran. Besides, there existed at least 72 hours intervals between the two stages of the test. The laboratory ambient temperature was $25^{\circ} \mathrm{C}$ and the humidity was $35 \%$. The maximum oxygen intake was measured using a respiratory gas analyzer system (Ganshorn model), i.e., calibrated before each test. The Bruce test was also used in this analysis. Systolic Blood Pressure (SBP) and Diastolic Blood Pressure (DBP) values were collected before performing the test. SBP was determined by the appearance of Korotkoff sounds, while the point of disappearance of these sounds was considered as DBP. Furthermore, we analyzed the new numerical value obtained by subtracting the sum of SBP and DBP after exercise from rest, separately. Then, the study participants started the activity while listening to music or no music according to their session. Eventually, the research participants continued the Bruce test until exhaustion. Additionally, according to the research background, the loud sound of the music was considered to be 100 decibels. When the examined participants announced their inability to continue, the treadmill was stopped. The maximum heart rate was recorded immediately after the test. We calculated and registered their SBP and DBP as soon as they sat in the chair, then subtracted the sum of SBP and DBP after exercise from the rest separately and analyzed the new numerical value.

\begin{tabular}{ccc}
\hline Variables/Statistics Index & SD \pm Mean & Variation Range \\
\hline Age & $0.96 \pm 23.75$ & $20-25$ \\
\hline height & $6.60 \pm 179.7$ & $1.02-1.83$ \\
weight & $8.07 \pm 69.26$ & $64.79-73.74$ \\
\hline BMI & $1.39 \pm 21.49$ & $21.1-24.6$ \\
\hline VO2 max & $3.63 \pm 43.14$ & $41.13-45.15$ \\
\hline
\end{tabular}


Table 2. Descriptive statistics results

\begin{tabular}{|c|c|c|c|c|c|c|}
\hline \multirow{2}{*}{ Variables } & \multirow{2}{*}{ Factors } & \multirow{2}{*}{ Mean } & \multirow{2}{*}{ Std. Deviation } & \multirow{2}{*}{ Std. Error of Mean } & \multicolumn{2}{|c|}{$95 \% \mathrm{Cl}$} \\
\hline & & & & & Lower & Upper \\
\hline \multirow{5}{*}{ No music } & HR max & 186.2 & 12.82 & 3.311 & 179.1 & 193.3 \\
\hline & VO2 max & 43.14 & 3.636 & 0.938 & 41.13 & 45.15 \\
\hline & SBP & 3.667 & 1.759 & 0.454 & 2.692 & 4.641 \\
\hline & DBP & 0.766 & 0.460 & 0.119 & 0.511 & 1.022 \\
\hline & Time to exhaustion & 12.79 & 2.058 & 0.531 & 11.65 & 13.93 \\
\hline \multirow{5}{*}{ Slow music } & HR max & 179.7 & 15.06 & 3.89 & 171.4 & 188.1 \\
\hline & VO2 max & 41.74 & 5.027 & 1.298 & 38.96 & 44.53 \\
\hline & SBP & 2.373 & 1.422 & 0.367 & 1.586 & 3.161 \\
\hline & DBP & 0.666 & 0.523 & 0.135 & 0.376 & 0.956 \\
\hline & Time to exhaustion & 12.44 & 1.006 & 0.259 & 11.88 & 12.99 \\
\hline \multirow{5}{*}{$\begin{array}{l}\text { Progressive } \\
\text { music }\end{array}$} & HR max & 191.8 & 5.158 & 1.332 & 188.9 & 194.7 \\
\hline & VO2 max & 48.19 & 5.334 & 1.377 & 45.23 & 51.14 \\
\hline & SBP & 4.38 & 1.655 & 0.427 & 3.46 & 5.29 \\
\hline & DBP & 1.067 & 0.703 & 0.181 & 0.677 & 1.456 \\
\hline & Time to exhaustion & 14.64 & 1.605 & 0.414 & 13.76 & 15.53 \\
\hline
\end{tabular}

Graph pad prism was used to analyze the obtained data. The Shapiro Wilk test was used to check the normal distribution of data. Accordingly, if the achieved data were normal $(P>0.05)$ for more than two groups, a one-way Analysis of Variance (ANOVA) was performed, followed by multiple comparisons with the Tukey's HSD post hoc test. If the data were not normally distributed, the analogous non-parametric Kruskal-Wallis test was used to look at intergroup differences (more than two groups). For all statistical tests, a significance level of $\mathrm{P}<0.05$ was considered.

\section{Ethical considerations}

Individuals provided signed informed consent forms at the onset of the study after being informed of all the conditions, including risks and benefits. To get acquainted with the test conditions and activity on the treadmill, a briefing session was carried out. By asking about their medical records, it was also ensured that none of the study participants had hearing impairments. Furthermore, the subjects could cancel the test at any time if desired.

\section{Results}

ANOVA was used to assess the time to exhaustion. Moreover, the Kruskal-Wallis test was used to determine the data on maximum heart rate, maximal oxygen intake, SBP, and DBP. The inter-group results indicated that listening to slow music, compared to non-musical situations reduced the difference of SBP and DBP, maximum oxygen consumption, HR max, and time of exhaustion; however, this statistic was not significant $(P=0.134$, $\mathrm{P}=0.993, \mathrm{P}=0.999, \mathrm{P}=0.160, \mathrm{P}=0.819$, respectively). Additionally, listening to progressive music, compared to the non-musical state, insignificantly increased the difference between SBP and DBP and HR max $(P=0.735$, $\mathrm{P}=0.999, \mathrm{P}=0.496$, respectively). However, it caused a significant increase in maximum oxygen consumption and time of exhaustion ( $\mathrm{P}=0.043, \mathrm{P}=0.008$ respectively). Listening to progressive music, compared to slow music caused a significant increase in the difference between SBP, maximum oxygen consumption, HR max, and time to exhaustion $(P=0.004, P=0.009, P=0.002, P=0.001$, re- 
spectively); however, this increase in the difference of DBP was not significant $(P=0.253)$ (Table 2).

\section{Discussion}

The present study results conveyed a mixed message. This study revealed that external intervention (music) affected the difference in SBP. Each of these variables was examined in a separate hypothesis. The collected results signified that according to the significant values reported from the difference of SBP, music significantly impacted the state of progressive music, compared to slow music. However, this finding was not significant in the other two conditions, as follows: slow music, compared to non-music mode, and progressive music, compared to non-music mode. According to the P-values reported in the difference DBP, there was no significant difference between the external intervention in the following conditions: slow music, compared to non-music mode, progressive music, compared to non-music mode, and progressive music, compared to slow music. Studies on the effects of music on blood pressure during exercise reported conflicting results; however, these studies are limited. The present study data were inconsistent with those of Birnbaum et al. [11]. They reported that listening to music during exercise did not significantly change SBP and DBP in non-athletic men. Szmedra Bacharach also noted that listening to music reduced blood pressure during exercise [15]. Again, these contradictory results may be attributed to differences in the type of music, the conditions of the subjects, as well as the type, duration, and severity of the protocol used during the test. Given that the Sympathetic Nervous System (SNS) plays a key role in regulating blood pressure, and stimulation through the auditory system, can affect the SNS and cause changes in blood pressure [16].

Another goal of the present study was to investigate the effects of external interfe rence (music) on maximum oxygen consumption. The obtained results declared that music provides a significant effect on two conditions; progressive music, compared to slow music, and progressive music, compared to non-musical status. However, this difference was not significant in the state of slow music compared to $n$ on-music mode. These findings were in line with the reports by Simpson et al., Birnbaum et al., Bacon C et al., as well as Karageorghis and associates. They all mentioned that stimulus music presented a significant effect on the maximum amount of consumed oxygen [11, 17-19]. Bacon's study examined the effect of music on maximal oxygen consumption; they concluded that fast music had a significant effect on VO2 max results compared to slow music and non-music status $[17,20]$. Birnbaum et al. reported that listening to fast mus ic significantly increased oxygen consumption; however, slow music did not significantly change oxygen consumption during exercise [11]. VO2 max is a point where the body is no longer able to consume oxygen despite increased exercise intensity, and individuals who are better prepared have higher oxygen consumption and can exercise more intensely. Therefore, these plateau in the volume of oxygen consumption during a series of maximum training with constant working intensity or training with increasing work intensity, indicate the maximum oxygen consumption. The amount of VO2 max is determined by the activity of 3 vital organs. The first is the respiratory system, which transports oxygen from the atmosphere to the lungs and then to the bloods tream. The cardiovascular system, which moves blood and distributes it to various parts of the body, is the second. The muscle system, which requires oxygen to derive energy from carbohydrate and fat intake, is the third one. Furthermore, factors that affect the determination of the maximum amount of oxygen consumed included the following: cardiac output, i.e., the amount of blood pumped per the contraction of the ventricle, the body's blood-oxygen carrying capacity (the hemoglobin of red blood cells), exercise skeletal muscle volume, the ability of muscle fibers to consume oxygen, and the last factor is genetics (40\%-90\%). Additionally, listening to progressive music during exercise significantly increases heart rate and according to Equation 1 , the cardiac output also increased.

\section{1) $Q=H R(b p m) S V(m L)$}

As a result, this increase may affect the amount of $\mathrm{VO} 2$ max, and the in dividual will later reach the VO2 max plateau. As mentioned, these contradictory results may be due to differences in numerous characteristics, such as cultural diversity, racial diversity, linguistic diversity, musical theme, weather conditions, the gender of the subjects, the level of physical fitness of the subjects, lack of control over some of the effective physical conditions, and even lack of control over the mental and the psychological conditions of the subjects.

The achieved findings revealed that music plays a major role in heart rate in the situation of progressive music vs. slower music; however, this difference was not significant in these conditions: slow music, compared to non-music mode, and progressive music, compared to non-music mode. Barwood et al. also reported that stimulant intervention increased heart rate, compared to non-stim ulating and control interventions [7]. Tiev et al. also examined the effects of music on cardiovas- 
cular responses. They reported that music significantly increased heart rate. Both studies were consistent with the present study [13]. Brownley et al. reported that music presented no significant effect on subjects' heart rate [21]. According to previous discussions, music can affect the sympathetic and parasympathetic nervous systems through the auditory system; consequently increasing and decreasing the heart rate. However, the style of music and activity both influence heart rate. Moreover, different types of music can elicit different responses. Because of its sedative properties, slow music decreases sympathetic nervous system activity and heart rate; however, fast music stimulates the sympathetic nervous system, which raises the heart rate due to its stimulating and emotional qualities.

The present study findings revealed that music also signific antly influences the time to exhaustion in two statuses; progressive music, compared to the non-music situation, and progressive music, compared to slow music. However, this difference was not significant in the case of slow music, compared to non-music conditions. The effects of music on maximal and sub-maximal exercise performance in non-athletic boys were investigated in one study. Subsequently, the findings revealed that listening to various styles of music while performing maximum performance in the Bruce test had no major effect on the time to exhaustion [16]. Macone et al. documented that listening to music during exercise provided no significant effect on time exhaustion [22]. Crust in 2004, Copeland, and Franks reported that music is ineffective in the time to exhaustion; therefore, these studies' data were inconsistent with those of the present study [23, 24]. Jorn Lemmer investigated the effects of the external intervention on the time to exhaustion and return to its resting state after continuous training in male athletes. The relevant results indicated that the time to exhaustion in exercising significantly increased with music conditions with moderate rhythm [25]. Szabo e al., Elliott Dave et al., and Nakamura et al. also found that music delays fatigue. Besides, researchers found that muscle tension changes with different types of music; thus, slow music may reduce muscle activity during exercise. Therefore, it increases the motivation of the individual to do the exercise and allows him to work longer [26-28].

\section{Conclusion}

Listening to progressive music during exercise can affect physiological factors and performance, according to the findings of this research. Progressive music raises the athlete's maximum oxygen intake and time to ex- haustion, enhancing incentive to exercise and delaying fatigue; thus, this can be a significant factor in exercise training to improve the capacity to receive oxygen and activity time, during exercise. Slow music, on the other hand, induces a relaxed state during exercise, which decreases heart rate and blood pressure. As a result, individuals with hypertension who exercise while listening to soft music will decline their blood pressure.

\section{Ethical Considerations}

\section{Compliance with ethical guidelines}

The related Ethics Committee of the Department of Physical Education and Sport Science, University of Tehran approved the present study.

\section{Funding}

This research did not receive any grant from funding agencies in the public, commercial, or non-profit sectors.

\section{Authors' contributions}

All authors equally contributed to preparing this article.

\section{Conflict of interest}

The authors declared no conflict of interest.

\section{Acknowledgments}

I'd like to express my sincere thanks to those who volunteered for this report.

\section{References:}

[1] Noble BJ, Robertson RJ. Perceived exertion. Champaign: Human Kinetics; 1996. https://books.google.com/ books?id=cdCONGFsZ9oC\&dq

[2] Bateman A, BaleJ,Sporting sounds: Relationships between sportand music. London: Routledge; 2008. [DOI:10.4324/9780203887974]

[3] Karageorghis Cl, Priest D-L. Music in the exercise domain: A review and synthesis (Part I). International Review of Sport and Exercise Psychology. 2012; 5(1):44-66. [DOI:10.1080/175098 4X.2011.631027] [PMID] [PMCID]

[4] Vaez Mousavi SMK, Nikbakhsh R. The impact of sensory and music deprivation on perception of pressure and temperament during exercise (Persian)]. Olympic Quarterly. 1997; 5(1-2):55-61. http:// ensani.ir/fa/article/308674

[5] Karageorghis $\mathrm{Cl}$, Terry PC. The psychophysical effects of music in sport and exercise: A review. Journal of Sport Behavior. 1997; 20(1):54-68. https://www.researchgate.net/publica- 
tion/49401604_The_psychophysical_effects_of_music_in_sport_ and_exercise_A_review

[6] Thakare AE, Mehrotra R, Singh A. Effect of music tempo on exercise performance and heart rate among young adults. International Journal of Physiology, Pathophysiology and Pharmacology. 2017; 9(2):35-9. [PMID] [PMCID]

[7] Barwood MJ, Weston NJV, Thelwell R, Page J. A motivational music and video intervention improves high-intensity exercise performance. Journal of Sports Science \& Medicine. 2009; 8(3):435-42. [PMID] [PMCID]

[8] McCraty R, Barrios-Choplin B, Atkinson M, Tomasino D. The effects of different types of music on mood, tension, and mental clarity. Alternative Therapies in Health and Medicine. 1998; 4(1):75-84. [PMID]

[9] Pujol TJ, Langenfeld ME. Influence of music on Wingate Anaerobic Test performance. Perceptual and Motor Skills. 1999; 88(1):292-6. [DOI:10.2466/pms.1999.88.1.292] [PMID]

[10] Beshai S, Watson LM, Meadows TJS, Soucy JN. Perceptions of cognitive-behavioral therapy and antidepressant medication for depression after brief psychoeducation: Examining shifts in attitudes. Behavior Therapy. 2019; 50(5):851-63. [DOI:10.1016/j. beth.2019.01.001] [PMID]

[11] Birnbaum L, Huschle B, Boone T. Cardiovascular responses to music tempo during steady-state exercise. Journal of Exercise Physiology. 2009; 12(1):50-7. http://fisioterapi.polanka.ac.id/wp-content/uploads/2018/03/CARDIOVASCULAR-RESPONSES-TO-MUSIC-TEMPO.pdf

[12] Koç H, Curtseit T. The effects of music on athletic performance. Ovidius University Annals, Series Physical Education \& Sport/Science, Movement \& Health. 2009; 9(1):43-7. https:// web.p.ebscohost.com/abstract?direct=true\&profile=ehost\&scope=sit e\&authtype=crawler\&jrnl=\%3d53974740

[13] Tiev M, Manire SA, Robert JR, Barbara W. Effect of music and dialogue on perception of exertion, enjoyment, and metabolic responses during exercise. International Journal of Fitness. 2010; 6(2):45-52. https://web.b.ebscohost.com/abstract?direct=true\&pr ofile $=3 \mathrm{~d} 52722029$

[14] Barzegar H, Vosadi E, Soori R, Akbarnejad A. [Effect of music on athlete metabolic responses during incremental exercise (Persian)]. Medical Sciences Journal of Islamic Azad University Tehran Medical Branch. 2014; 24(3):153-8. http://tmuj.iautmu.ac.ir/article-1-835-fa.html

[15] Szmedra L, Bacharach DW. Effect of music on perceived exertion, plasma lactate, norepinephrine and cardiovascular hemodynamics during treadmill running. International Journal of Sports Medicine. 1998; 19(1):32-7. [DOI:10.1055/s-2007-971876] [PMID]

[16] Meis JK. Modification of perceived enjoyment, exertion and performance among novice and experienced exercisers: A cognitivebehavioral approach to perceptual change [PhD dissertation]. Tallahassee: Florida State University; 2003. https://www.proquest.com/ openview/9568998294c0da472e5150f8a0aeeeac/1?pq-origsite=g scholar \&cbl=18750\&diss $=y$

[17] Bacon C, Myers T, Karageorghis Cl. Effect of movement-music synchrony and tempo on exercise oxygen consumption. Manuscript submitted for publication. 2008. https://scholar.google.com/ scholar?hl=en\&as_sdt=0\%2C5\&q+\&btnG=

[18] Karageorghis Cl, Jones L, Stuart DP. Psychological effects of music tempi during exercise. International Journal of Sports Medicine. 2008; 29(7):613-9. [DOI:10.1055/s-2007-989266] [PMID]
[19] Simpson SD, Karageorghis Cl. The effects of synchronous music on 400-m sprint performance. Journal of Sports Sciences. 2006; 24(10):1095-102. [DOI:10.1080/02640410500432789] [PMID]

[20] Eliakim M, Meckel Y, Nemet D, Eliakim A. The effect of music during warm-up on consecutive anaerobic performance in elite adolescent volleyball players. International Journal of Sports Medicine. 2007; 28(04):321-5. [DOI:10.1055/s-2006-924360] [PMID]

[21] Brownley KA, McMurray RG, Hackney AC. Effects of music on physiological and affective responses to graded treadmill exercise in trained and untrained runners. International Journal of Psychophysiology. 1995; 19(3):193-201. [DOI:10.1016/0167-8760(95)00007-F]

[22] Macone D, Baldari C, Zelli A, Guidetti L. Music and physical activity in psychological well-being. Perceptual and Motor Skills. 2006 103(1):285-95. [DOI:10.2466/PMS.103.5.285-295] [PMID]

[23] Crust L, Clough PJ. The influence of rhythm and personality in the endurance response to motivational asynchronous music. Journal of Sports Sciences. 2006; 24(2):187-95. [DOI:10.1080/02640410500131514] [PMID]

[24] Copeland BL, Franks BD. Effects of types and intensities of background music on treadmill endurance. The Journal of Sports Medicine and Physical Fitness. 1991; 31(1):100-3. [PMID]

[25] Lemmer B. Effects of music composed by Mozart and Ligeti on blood pressure and heart rate circadian rhythms in normotensive and hypertensive rats. Chronobiology International. 2008; 25(6):971-86. [DOI:10.1080/07420520802539415] [PMID]

[26] Szabo A, Small A, Leigh M. The effects of slow-and fast-rhythm classical music on progressive cycling to voluntary physical exhaustion. Journal of Sports Medicine and Physical Fitness. 1999, 39(3):220-5. [PMID]

[27] Elliott D, Carr S, Orme D. The effect of motivational music on submaximal exercise. European Journal of Sport Science. 2005; 5(2):97-106 [DOI:10.1080/17461390500171310]

[28] Nakamura PM, Pereira G, Papini CB, Nakamura FY, Kokubun E. Effects of preferred and nonpreferred music on continuous cycling exercise performance. Perceptual and Motor Skills. 2010; 110(1):257-64. [DOI:10.2466/pms.110.1.257-264] [PMID] 
This Page Intentionally Left Blank 